\title{
Efficacy and Safety of Vildagliptin Compared to Sulphonylurea in Patients with Type 2 Diabetes during Fasting in Ramadan
}

\author{
MF PATHAN ${ }^{\mathrm{a}}$, MF AMIN $^{\mathrm{b}}, \mathrm{F}$ AFSANA $^{\mathrm{c}}$, MSA RAHIM $^{\mathrm{d}}$, MJA SARKER $^{\mathrm{e}}$, \\ TMALI $^{\mathrm{f}}$, M MM RAHMAN', F HASAN $^{\mathrm{h}}$
}

Summary:

The objective of this study is to evaluate the efficacy and safety of vildagliptin compared to sulphonylurea (SU) in Type 2 Diabetes during Fasting in Ramadan. This was a prospective, observational cohort study, which enrolledpatients from Bangladesh. Patients aged $\geq 18$ years with T2DM and HbA1C $\leq 8.5 \%$ were treated with vildagliptin or $S U$ as add-on to metformin or as monotherapy for 16 weeks.The primary outcome of interest was to compare the proportion of patients with $\geq 1$ hypoglycemic event(s) (HE) during fasting between the vildagliptin and SU groups. Changes in HbA1c, body weight and treatment adherence were also measured. Of the 100 patients enrolled, 97completed the study and 3 patients discontinued prematurely. Patients experiencing $\geq 1 \mathrm{HE}(\mathrm{s})$ were fewer in the vildagliptingroup compared with $S U$ group

\section{Introduction:}

Ramadan is a holy month (the ninth month of the Islamic calendar) for the Muslims worldwide and fasting from

a. Prof. Md. Faruque Pathan, Department of Endocrinology, BIRDEM Hospital, Bangladesh.

b. Dr. Md. Feroz Amin, Department of Endocrinology, BIRDEM Hospital, Bangladesh.

c. Dr. Faria Afsana, Department of Endocrinology, BIRDEM Hospital, Bangladesh.

d. Dr. Md. Saghir Abdur Rahim, Department of Endocrinology, BIRDEM Hospital, Bangladesh.

e. Dr. Md. Jahangir Alam Sarker, Department of Endocrinology, BIRDEM Hospital, Bangladesh.

f. Dr. Tanvir Mobarak Ali, Novartis Bangladesh Limited, Bangladesh.

g. Dr. Md. Mahfuzur Rahman, Novartis Bangladesh Limited, Bangladesh.

h. Dr. Farzana Hasan, Novartis Bangladesh Limited, Bangladesh.

Address of Correspondence: Professor Md. Faruque Pathan, Professor and Head, Department of Endocrinology, BIRDEM Hospital, 122, Kazi Nazrul Islam Avenue, Shahbagh, Dhaka1000, Bangladesh, Contact No.: +8801713032888, E-mail: pathan279@yahoo.com

Received: 31 February, 2014

Accepted: 28 April, 2015
(4.3\% vs. 8.2\%; $p=0.678$ ). The reduction in $\mathrm{HbA1c}$ was $0.1 \%$ with vildagliptin from a baseline of $7.1 \%$,however, there was no changewith $S U$ from a baseline of $7.2 \%$ (between-treatment difference: "0.1\%; $p=0.600)$. A gain of $0.35 \mathrm{~kg}$ and $0.08 \mathrm{~kg}$ was seen with vildagliptinand SUs treatment, respectively. Overall, the incidence of adverse events was similar betweenthe vildagliptin and SU groups (23.4\% vs. 20.4\%) with no new safety signals.

The treatment with vildagliptin was associated with fewer hypoglycemic events compared with $S U$ and was well tolerated in Muslim T2DM patients fasting during Ramadan.

Keywords: DPP-4 inhibitor, Hypoglycemia, Ramadan, Type 2 Diabetes Mellitus, Vildagliptin, Sulphonylurea.

(J Banagladesh Coll Phys Surg 2015; 33: 120-125)

predawn to after sunset is widely practiced ${ }^{1}$. Depending on the geographical location and time of the year, fasting can last from 11 to 20 hours a day. As fasting should not create excessive hardships, certain individuals who are ill are exempted from fasting. However, a majority of Muslim patients with type 2 diabetes mellitus (T2DM) do not consider themselves ill, and are ardent about their Ramadan fasting, despite the complications such as hyperglycemia and hypoglycemia, diabetic ketoacidosis, dehydration, and thrombosis. ${ }^{2,3}$ The Population-based Epidemiology of Diabetes and Ramadan (EPIDIAR)study showed that there was a 7.5fold increase in the risk of severe hypoglycemic events (HEs)and a 5-fold increase in hospitalization due to hypoglycemia in fasting patients with T2DM. ${ }^{4}$ Therefore, the risk of hypoglycemia is to be taken into account while choosing or maintaining an antidiabetic agent during Ramadan.

Current Ramadan guidelines (South Asian guidelines for the management of endocrine disorders in Ramadan) recommend individual assessments, investigations, counseling,as well as necessary changes in lifestyle, diet, and medication for patients fasting during 
Ramadan. ${ }^{5,6}$ There is no consensus about the most appropriate oral antihyperglycemic agent(s) for patients with T2DM during Ramadan, as there is limited data assessing the efficacy and safety of these agents during Ramadan. Sulphonylureas (SUs) and glinides are widely used as antihyperglycemic agents due to their wellestablished clinical profile and lowcost;however, they are associated with an increased risk of hypoglycemiaand weight gain. ${ }^{7,8}$ Dipeptidyl peptidase4 (DPP-4) inhibitors are important substitutes for SUs in patients with T2DM during fasting owing to their glucose-dependent mechanism of action, efficacy, and tolerability. ${ }^{9}$ Vildagliptin is a potent and selective DPP4 inhibitor that improves glycemic control by increasing the a- and b-cell responsiveness to glucose. ${ }^{10}$ It significantly reduces the risk of hypoglycemiacompared with sulphonylurea. ${ }^{11}$

The VIldagliptInexpe Rience compared wiThsulphonyl Ureasobs Erved (VIRTUE) study was conducted in 12 countries of the Middle East and Asia including Bangladesh. The results of the study showed thattreatment with vildagliptin was associated with significantly fewer patients experiencing hypoglycemia compared with SUs in fasting Muslim patients with T2DM. ${ }^{11}$ As there is a dearth of literature on patients with T2DM fasting during Ramadan in Bangladesh,the present study assessed the proportion of patients with $\geq 1 \mathrm{HE}$ (Hypoglycemic Event) receiving vildagliptin or $\mathrm{SU}$ as add-on to metformin or as monotherapy during fastingin Ramadan.

\section{Methods:}

Study design and patient population

This was a prospective, observational cohort study, which included patients aged $\geq 18$ years with duration of T2DM for more than 12 months before the start of Ramadan fasting (HbA1c $\leq 8.5 \%$ measured within 6 weeks before the study entry).Patients were treatedwith vildagliptin or SUs as add-on to metformin or as monotherapy for at least 4 weeks, but not more than 3 years before fasting commenced.

Patients were excluded if they had contraindications to the study medicationor if they required three or more oral antidiabetesdrugs or insulin therapy at the time of the study entry. During the observational period of approximately 16 weeks, data from at least two routine clinic visits were recorded for each patient at baseline (6 weeks before the start of fasting) and at the end of the study (within 6 weeks after the end of fasting). At both the visits,HbA1cwas estimated. Patients were provided a diary / log book during their fasting period and were asked to record the diabetes treatment time points, blood glucose measurements and hypoglycemia symptoms. This data was used to assess patient adherence to prescribed dosages and timing of doses while fasting.Information was collected from Patient log book supplied which included total day of fasting or missing of fasting, daily anti-diabetic agent intake, hypo or any other adverse events, confirmed hypoglycemia and how they overcome.

Data collection was also permitted during the observational period if the patient made an interim visit during the fasting period.A written informed consent for the collection and use of data was obtained from all the participants, and the study was conducted in accordance with the guidelines for Good Pharmacoepidemiological Practices, national requirements and regulations, in line with the ethical principles laid down in the Declaration of Helsinki. Ethical clearance for the study was received from BADAS Ethical Review Committee.

\section{Study outcome assessments}

The primary objective was to assess the proportion of patients who experienced at least one HE during the fasting period.HEs were categorized by investigators as mild or grade 1 , defined as any reported symptoms by the patient and/or any blood glucose measurement $<3.9 \mathrm{mmol} / \mathrm{L}$ (70 $\mathrm{mg} / \mathrm{dL}$ ), and severe or grade 2, defined as the need for third-party assistance. Secondary objectives included assessment of changes in body weight and $\mathrm{HbA1c}$ from pre-fasting baseline to the study endpoint and treatment adherence (proportion of patients who did not miss more than $20 \%$ of the prescribed medication doses during Ramadan).Overall safety was assessed by monitoring and recording adverse events (AEs) and serious AE (SAEs). Hypoglycemia-related symptoms or any other AEs were recorded by the patient in the paper diary or log book. If a patient did not record HEs or glucose readings, the number of HEs experienced was recorded by the physician at the end of observational period, as recalled by the patient.

\section{Statistical analysis}

For the primary assessment variable, based on $90 \%$ power and a two-sided significance level of 0.05 , a sample size of 35 patients per group would be sufficient to detect a $40 \%$ difference between the proportions of patients experiencing $\geq 1 \mathrm{HE}(\mathrm{s})$, tested with a two-group continuity corrected chi-squared test. The change in 
HbA1c from baseline was computed and summarized.The difference estimate is based on the published incidences of HEs for patients receiving SU (60\%) and vildagliptin (d"20\%) $)^{12}$.The primary study variable was analyzed using a two-sided Fisher's exact test performed on data from all patients who received atleast one dose of the study medication at the beginning of Ramadan and had at least one efficacy assessment after the start of fasting (primary analysis set, [PAS]). Apart from HbA1c assessments, other assessments were performed on the safety population, consisting of all patients who received at least one dose of the study medication at the beginning of Ramadan and had at least one safety assessment. HbA1c and body weight data wereanalyzed using the last observation carried forwardapproach. Data were analyzed by DATAMAP GmbH, Freiburg, Germany using SAS $^{\circledR}$, Release 9.3 (SAS Institute Inc., Cary, NC).

\section{Results:}

Of the 100 patients enrolled from Bangladesh, 97 patients completed the study andthree patients discontinued prematurely.A total of 50 patients were treated with vildagliptinand 49 patients were treated with an SU. One patient was neither treated with vildagliptin nor with an SU, and therefore was excluded from the study.In the vildagliptingroup, three (6.0\%) patients were excluded from the PAS and safety set due to withdrawal before the start of Ramadan. The key demographics and clinical background characteristics were broadly similar in both vildagliptin and SU groups except for body weight and body mass index (BMI) (Table I).The mean age of patientswas 50.3 years, $55.2 \%$ were male, mean body weight was $69.1 \mathrm{~kg}$, and mean BMI was $26.7 \mathrm{~kg} / \mathrm{m}^{2}$. The mean baseline $\mathrm{HbA} 1 \mathrm{c}$ was $7.1 \%$ in the vildaglipt in group and $7.2 \%$ in the SU group.The mean weight of patients in the vildagliptingroup was higher $(71.5 \mathrm{~kg}$ ) compared with patients in the SU group (66.8 kg), which led to the difference in BMI as well.

A majority of the patients were receiving metformin in combination with the study medication: $97.9 \%(n=46 /$ 47) in the vildagliptin group and $83.7 \%(n=41 / 49)$ in the SU group.Median daily dose of vildagliptinwas $100 \mathrm{mg}$ andof SUs were: glimepiride $1 \mathrm{mg}(\mathrm{n}=24)$, gliclazide70 $\mathrm{mg}(\mathrm{n}=20)$, glipizide $7.5 \mathrm{mg}(\mathrm{n}=2)$ and glibenclamide 5 $\mathrm{mg}(\mathrm{n}=1)$. In both the treatment groups, the mean exposure during Ramadan (vildagliptin, 30 days; SU, 30 days) and the mean number of days fasting (vildagliptin, 27.9 days; SU, 29.4 days) were similar.

\section{Frequency of HEs}

Numerically fewer patients experienced $\geq 1 \mathrm{HE}$ (s) with vildagliptin ( $n=2 / 47,4.3 \%)$ compared with those receiving SUs ( $n=4 / 49,8.2 \%$ ), resulting in a $50.0 \%$ relative risk reduction (odds ratio [OR] $=0.5 ; 95 \%$ CI: 0.087,2.869; $\mathrm{p}=0.678$; Table III). All the patients experiencing hypoglycemia during fasting reported a single $\mathrm{HE}$ in both the groups.No patient reported a grade $2 \mathrm{HE}$.

Table-I

Patient demographics and baseline characteristics

\begin{tabular}{lccc}
\multicolumn{4}{c}{ Patient demographics and baseline characteristics } \\
Variable & Vildagliptinn $=47$ & Sulphonylurean $=49$ & Total \\
\hline Age, years & $49.8(10.0)$ & $50.7(10.1)$ & $50.3(10.0)$ \\
$<65, \mathrm{n}(\%)$ & $45(95.7)$ & $47(95.9)$ & $92(95.8)$ \\
Male, $\mathrm{n}(\%)$ & $14(29.8)$ & $39(79.6)$ & $53(55.2)$ \\
Weight, kg & $71.5(12.4)$ & $66.8(9.2)$ & $69.1(11.1)$ \\
BMI, kg/m² & $28.1(3.8)$ & $25.4(3.1)$ & $26.7(3.7)$ \\
Duration of T2DM, years & $5.9(5.0)$ & $4.5(3.2)$ & $5.2(4.2)$ \\
HbA1c, \% & $7.1(0.7)$ & $7.2(0.8)$ & $7.1(0.8)$ \\
Diabetes medication changed for fasting during Ramadan, n (\%) & \\
No & $43(91.5)$ & $40(81.6)$ & $83(86.5)$ \\
\hline
\end{tabular}

Values are mean \pm SD unless indicated otherwise. BMI, body mass index; HbA1c, hemoglobin A1c; SD, standard deviation; T2DM, type 2 diabetes mellitus. 


\section{Table-II}

\begin{tabular}{|c|c|c|}
\hline \multicolumn{3}{|c|}{$\begin{array}{l}\text { Adverse events occurring in any treatment } \\
\text { group (safety set) }\end{array}$} \\
\hline Event, n (\%) & $\begin{array}{c}\text { Vildagliptin } \\
\mathrm{n}=47\end{array}$ & $\begin{array}{c}\text { Sulphonylurea } \\
\mathrm{n}=491\end{array}$ \\
\hline Any AE & $12(25.5)$ & $8(16.3)$ \\
\hline \multicolumn{3}{|c|}{$\begin{array}{l}\text { Most common AEs by } \\
\text { preferred term* }\end{array}$} \\
\hline Pyrexia & $4(8.5)$ & $2(4.1)$ \\
\hline Asthenia & $2(4.3)$ & $0(0.0)$ \\
\hline Hypoglycemia & $2(4.3)$ & $4(8.2)$ \\
\hline Headache & $2(4.3)$ & $0(0.0)$ \\
\hline Restlessness & $2(4.3)$ & $0(0.0)$ \\
\hline Peptic ulcer & $0(0.0)$ & $2(4.1)$ \\
\hline
\end{tabular}

Table-III

Number of patients with hypoglycemic events (HEs) during Ramadan fasting

\begin{tabular}{lcc} 
Event & $\begin{array}{c}\text { Vildagliptin } \\
(\mathrm{n}=47)\end{array}$ & $\begin{array}{c}\text { Sulphonylurea } \\
(\mathrm{n}=49)\end{array}$ \\
\hline $\begin{array}{l}\text { Patients with } \geq 1 \\
\text { hypoglycemic } \\
\text { events }\end{array}$ & 2 & 4 \\
\hline$P=0.678 *$ & \\
*Fishers exact test.
\end{tabular}

Table-IV

Mean change in HbA1c pre- to post-Ramadan in the vildagliptin and $S U$ groups

\begin{tabular}{lcc} 
Event & $\begin{array}{c}\text { Vildagliptin } \\
(\mathrm{n}=47)\end{array}$ & $\begin{array}{c}\text { Sulphonylurea } \\
(\mathrm{n}=49)\end{array}$ \\
\hline $\begin{array}{l}\text { Mean change in } \\
\text { HbA1c from }\end{array}$ & -0.1 & 0 \\
baseline (\%) & \\
\hline$P=0.600 *$ \\
*Two sample $t$-test. Last observation carried forward.
\end{tabular}

\section{HbA1c and body weight changes}

At the end of the study, the mean HbA1c level decreased by $0.1 \%$ in the vildagliptingroup from a pre-fasting baseline of 7.1\%; however,no change was seen in the SU groupfrom a pre-fasting baseline of $7.2 \%$ (“ $0.1 \%$ between-treatment difference; $\mathrm{p}=0.600$; Table IV.). Weight changes from pre- to post-Ramadan were 0.35 $\mathrm{kg}$ with vildagliptinversus $0.08 \mathrm{~kg}$ with $\mathrm{SUs}$, with a mean between-treatment difference of $0.27 \mathrm{~kg}$, which was not significant $(\mathrm{p}=0.528)$.

Treatment adherence

Treatment adherence was high in both the treatment groups( $(98.0 \%)$ : 46 patients $(97.9 \%)$ in the vildagliptingroup and 48 patients (98.0\%) in the SU group. The number of doses (mean $\pm \mathrm{SD}$ ) missed during the fasting period was similar between the two groups with $1.4 \pm 4.6$ and $1.0 \pm 3.2$ in the vildagliptin group and the SU group, respectively.

\section{Safety and tolerability}

A total of 12 patients (25.5\%) in the vildagliptingroup and ten patients (16.3\%) in the SU group experienced AEs after the beginning of Ramadan fasting.Pyrexia (n $=4 ; 8.5 \%$ ) was the most common $\mathrm{AE}$ in the vildagliptingroup and hypoglycemia $(n=4 ; 8.2 \%)$ in the SU group (TableII).The majority of AEs were mild in severity.There were no SAEs and no deaths reported in both the groups and no patient discontinued the study due to AEs.

\section{Discussion:}

To our knowledge, this is a first prospective observational study conducted in Bangladeshin patientswith T2DM fasting during Ramadan. The results of this observational study showed that there is a $50 \%$ less risk for HEs in patients treated withvildagliptin compared with SUs. The present findings are consistent with the findings from the previous studies conducted on Muslims with T2DM fasting during Ramadan, which showed that the incidence of HEs was significantly lower in the vildagliptin group than in the SU group during Ramadan. ${ }^{12,13}$

The present results are also comparable with the results from other studies that used other DPP-4 inhibitor sitagliptin. In a study conducted in Egypt, Israel, Jordan, Lebanon, Saudi Arabia, and the UAE, the risk of symptomatic HE was significantly decreased with sitagliptincompared with SU (OR = 0.51; 95\% CI 0.34, 0.75). ${ }^{14}$ These resultsarealso supported withthe overall VIRTUE study findings, in which vildagliptin showed reduction in HEs compared with SU (5.4\% vs. 19.8\%, respectively). ${ }^{11}$ 
In the present study, a small increase in the mean body weight from baseline was observed in both the vildagliptin and SU groups, which was statistically not significant, as people of Bangladesh take food with more calorie value because of their dietary habit during Ramadan period. A slightimprovement in mean HbA1c was observed during the study period for the vildagliptingroup, whereas in the SU group, the mean HbA1c increased slightly. The improvement in HbA1c was smaller compared with thefindings fromthe VECTOR study which may be due to lowHbA1c at baseline compared with baseline HbA1c in the VECTOR study. ${ }^{13}$

Although hypoglycemia is a significant barrier to treatment adherence, ${ }^{15}$ the self-reported adherence in the present study was high in both the treatment groups (98\%).The present results are also comparable with the results from overall VIRTUE study. ${ }^{11}$

Interestingly, higher median daily dose of vildagliptin (100 mg daily) was compared with the lower median daily dose of SUs (glimepiride $1 \mathrm{mg}$ [n=24], gliclazide70 mg [n=20],glipizide $7.5 \mathrm{mg}$ [n=2], and glibenclamide 5 $\mathrm{mg}[\mathrm{n}=1])$. This could explain the lower incidence of HEs in the SU group. Higher frequency of HEs is expected with high doses of SUs compared with present median dose. Despite comparing with a very low dose of SU, vildagliptin therapy had numerically fewer HEs in this study.

Overall, the incidence rate of AE was similar between the vildagliptin and SU groups (23.4\% vs. 20.4\%) with no new safety signals. There were certain limitations of this study, such as small sample size, lack of diet, eating patterns, and exercise data. Being an observational study, there are certain inherent limitations and bias associated with it. Owing to the non-interventional nature of the study, the reported HEs did not require mandatory confirmation with blood glucose measurements, which can potentially under or overestimate the number of HEs due to subjectivity in perceiving symptoms of hypoglycemia. Other factors that limit inference of the results are varied eating practices among different cultures during Ramadan (two to four meals) and of recording of HEs in the diary due to recall bias. Despite the above-mentioned limitations, this study has enabled collection of data from a patient group in a real-lifesetting which could complement the findings of randomized, interventional clinical trials.
Patients with T2DM who wish to fast during Ramadan should be well prepared to make fasting as safe as possible. Medical counseling is important for diabetic patients concerning the potential risks if they decide to fast. Any modification in medication, if required, should be done before the start of Ramadan fasting in order to provide a stable glycemic control.

\section{Conclusion:}

Vildagliptin therapy was associated with numerically fewer patients experiencing hypoglycemia compared with SU therapy in fasting Muslim patients with T2DMfrom Bangladesh. Our findings suggest that vildagliptin is a suitable treatment option, particularly to reduce the risk of hypoglycemia during the long daytime fasting periods during Ramadan in Muslim patients with T2DM.

\section{Acknowledgment:}

The authors acknowledge all investigators at the participating centers and all patients for their commitment to the study that was supported by Novartis Pharma AG, Basel, Switzerland. Vasundhara Pathak, Novartis Healthcare Private Limited, India, provided the medical writing and editorial assistance for the manuscript. All authors participated in the development and reviewing of the manuscript and took full responsibility for the contents of the article. The study was funded by the Novartis Pharma Bangladesh.

\section{References:}

1. Rashed AH. The fast of Ramadan. BMJ 1992;304:521-2.

2. Almaatouq MA. Pharmacological approaches to the management of type 2 diabetes in fasting adults during Ramadan. Diabetes MetabSyndrObes 2012;5:109-19.

3. Ahmad J, Pathan MF, Jaleel MA, Fathima FN, Raza SA, Khan AK, Ishtiaq O, Sheikh A. Diabetic emergencies including hypoglycaemia during Ramadan. Indian J EndocrinolMetab2012;16(4):512-5.

4. Salti I, Bénard E, Detournay B, Bianchi-Biscay M, Le Brigand C, Voinet C, Jabbar A; EPIDIAR study group. A population-based study of diabetes and its characteristics during the fasting month of Ramadan in 13 countries: results of the epidemiology of diabetes and Ramadan 1422/2001 (EPIDIAR) study. Diabetes Care 2004;27(10):2306-11.

5. Bashir MI, Pathan MF, Raza SA, Ahmad J, Khan AK, Ishtiaq O, Sahay RK, Sheikh A, Zargar AH. Role of oral hypoglycemic agents in the management of type 2 diabetes mellitus during Ramadan. Indian J Endocrinol Metab 2012;16(4):503-7.. 
6. Al-Arouj M, Assaad-Khalil S, Buse J, Fahdil I, Fahmy M, Hafez S, Hassanein M, Ibrahim MA, Kendall D, Kishawi $\mathrm{S}$, et al. Recommendations for management of diabetes during Ramadan: update 2010. Diabetes Care 2010;33(8):1895-902.

7. Zargar A, Basit A, Mahtab H. Sulphonylureas in the management of type 2 diabetes during the fasting month of Ramadan. J Indian Med Assoc 2005;103(8):444-6.

8. Zargar AH, Siraj M, Jawa AA, Hasan M, Mahtab H. Maintenance of glycaemic control with the evening administration of a long acting sulphonylurea in male type 2 diabetic patients undertaking the Ramadan fast. Int J ClinPract2010;64(8):1090-4..

9. Baetta R, Corsini A. Pharmacology of dipeptidyl peptidase-4 inhibitors: similarities and differences. Drugs 2011;71:1441-67.

10. Dejager S, Schweizer A. Minimizing the risk of hypoglycemia with vildagliptin: Clinical experience, mechanistic basis, and importance in type 2 diabetes management. Diabetes Ther 2011; 2: 51-66.

11. Al-Arouj M, Hassoun AA, Medlej R, Pathan MF, Shaltout I, Chawla MS, Hristoskova S, Ditommaso S, Kadwa MY. The effect of vildagliptin relative to sufonylureas in
Muslim patients with type 2 diabetes fasting during Ramadan: the Virtue study 2013. Int J ClinPract 2013;67(10):957-63.

12. Devendra D, Gohel B, Bravis V, Hui E, Salih S, Mehar S, Hassanein M. Vildagliptin therapy and hypoglycaemia in Muslim type 2 diabetes patients during Ramadan. Int $\mathrm{J}$ ClinPract 2009;63(10):1446-50.

13. Hassanein M, Hanif W, Malik W, Kamal A, Geransar P, Lister N, Andrews C, Barnett A. Comparison of the dipeptidyl peptidase-4 inhibitor vildagliptin and the sulphonylureagliclazide in combination with metformin, in Muslim patients with type 2 diabetes mellitus fasting during Ramadan: results of the VECTOR study. Curr Med Res Opin 2011;27(7):1367-74.

14. Al-Sifri S, Basiounny A, Echtay A, Al Omari M, HarmanBoehm I, Kaddaha G, Al Tayeb K, Mahfouz AS, Al Elq A, Radican L, et al. 2010 Ramadan Study Group. The incidence of hypoglycaemia in Muslim patients with type 2 diabetes treated with sitagliptin or a sulphonylurea during Ramadan: a randomised trial. Int $\mathrm{J}$ ClinPract 2011;65(11):1132-40.

15. Amiel SA, Dixon T, Mann R, Jameson K. Hypoglycaemia in type 2 diabetes. Diabet Med 2008;25(3):245-54. 